\title{
Efficient Healthcare Framework for Senior Benefaction
}

\author{
M.Balamurugan, Periasamy J.K, C.Gnanapriya, K.Vijiyalakshmi, E.Vanitha
}

\begin{abstract}
The Internet of Things (IoT) has been broadly used to interconnect the accessible restorative assets and offer shrewd, dependable, and successful social insurance administration to the older people. Health checking for dynamic and helped living is one of the ideal models that can utilize the IoT preferences to improve the old way of life. An IoT engineering is altered for social insurance applications. The proposed design gathers the information and transfers it to the cloud where it is prepared and dissected. Criticism activities dependent on the examined information can be sent back to the user. The proposed framework plan theory target is having a low-control framework that can be worn during the day and be turbo charged as necessary. The framework is executed utilizing minimal effort segments, which makes it a moderate framework.
\end{abstract}

Keywords: Physical sensor, indoor positioning module, sensor interface circuit.

\section{EXISTING SYSTEM}

A portion of the gadgets which are available for checking wellbeing conditions are,

Wellness trackers-Expensive and doesn't anticipate any future alignments. It utilizes GPS for distinguishing area so indoor checking isn't possible. Heart Rate Monitor-They are substantial and are not portable. The starter execution assessment results have exhibited the productivity of the current framework - in spite of being an ease one. This makes the current framework a decent possibility for actualizing a wide arrangement of wearable social insurance frameworks. Our future work will incorporate how to verify the entrance of the information and will build up a versatile application that permits access of the information on handheld gadgets.

\section{CONSTRAINTS}

- Location following in the structure is troublesome.

- $\quad$ Emergency circumstances are difficult to oversee.

\section{PROPOSEDSYSTEM}

IoT wearable stages can be utilized to gather the required data of the client and convey such data remotely. It is handled or put away for following the ailments of the client. The built up an Android application that plans to record the information estimated (SBP-Systolic Blood Pressure, DBP Diastolic Blood Pressure and Heart Rate) by the biomedical

Revised Manuscript Received on October 15, 2019

M.Balamurugan, Computer Science and Engineering. Sri Sai Ram Engineering College, Chennai, Tamilnadu, India

Periasamy J.K, Computer Science and Engineering. Sri Sai Ram Engineering College, Chennai, Tamilnadu, India

C.Gnanapriya, Computer Science and Engineering. Sri Sai Ram Engineering College, Chennai, Tamilnadu, India

K.Vijiyalakshmi, Computer Science and Engineering. Sri Sai Ram Engineering College, Chennai, Tamilnadu, India

E.Vanitha, Computer Science and Engineering. Sri Sai Ram Engineering College, Chennai, Tamilnadu, India sensors that imparts by means of Bluetooth. Bluetooth LowEnergy(BLE) iBeacon which is broadly utilized for indoor situating is available at each room of the house. iBeacon transmits a Bluetooth low vitality sign to gather the presence inside its region. Ideal Node Selection calculation is utilized to give supportable area checking. The following sensor is cautioned when the client moves from its territory inclusion and it gets dormant. This Face following system helps in making productive medicinal services structure. The sensor information can be utilized in anticipating future sicknesses and sending periodical cautions to medicinal expert.

The framework proposes a versatile and adjustable IoT framework that can be utilized to gather the information expected to encourage the free living of senior and moved residents to improve their personal satisfaction. The proposed framework plan reasoning targets having a lowcontrol framework that can be worn during the day and be turbo charged as vital. The framework is intended to be light and agreeable to wear. Furthermore,the framework is actualized utilizing minimal effort parts, which makes it a moderate system. The framework is made out of four primary segments that are depicted as pursues.

\section{Heartbeat SENSOR :}

Heartbeat Sensor is a minimal effort, exceptionally little size an attachment and-play pulse sensor for Arduino and Arduino perfect sheets. It very well may be utilized by understudies, specialists, competitors, creators, and game and portable engineers who need to effectively join live pulse information into their projects. Pulse Sensor Amped includes enhancement and clamor crossing out hardware to the equipment.

\section{VIBRATION SENSOR:}

A fall recognition sensor is certainly not a restorative ready gadget you buy exclusively without anyone else. Or maybe, you add the fall identification sensor to a medicinal ready framework, either as a pendant or an initiated highlight on a cell phone. In view of this, you ought to pick a standout amongst other medicinal ready frameworks before you choose whether to include a fall location sensor. I tried the call reaction times, nature of the call focuses, speaker clearness and the fall discovery sensors. In the wake of contrasting expenses and the exhibition, the GreatCall Mobile Lively earned my pick for the best fall location sensor by and large since this administration furnishes the best restorative ready framework with the best fall discovery execution. 


\section{LIGHT SENSOR:}

This LM393 Photosensitive Light-Dependent Control Sensor Module is utilizing a top notch LM393 voltage comparator. Simple to introduce utilizing the delicate kind photosensitive obstruction sensor the comparator yield sign gives a perfect and great waveform. Driving capacity is $15 \mathrm{~mA}$ with the flexible potentiometer, it can change the brilliance of the light identified. Working voltage is $3.3 \mathrm{~V}$ to $5 \mathrm{v}$. Where yield is computerized switch yield. Since this module is delicate to the light, generally utilized for identifying the surrounding brilliance and light intensity. When there is no light or the light force can't arrive at the worth, DO yield is elevated level. At the point when light force over than the worth, the module DO yield is low level. The module computerized yield DO can be straightforwardly associated with the microcontroller, use microcontroller identifies high or low level, so that can distinguish the natural light force change.

\section{Driven:}

A LED light is a strong state lighting (SSL) gadget that fits in standard screw-in associations yet utilizes LEDs (light-radiating diodes) to create light. Driven lights are an all the more naturally agreeable option in contrast to radiant bulbs.

\section{AURDINO UNO MICROCONTROLLER:}

The sign speaking to the distinctive detected wonders are then adapted to be prepared for contribution to the microcontroller. This is accomplished using sensor interfacing hardware that changes over any simple sign originating from the sensors into the relating computerized group and plays out any further sign molding usefulness to guarantee similarity with the utilized Arduino UNO microcontroller.

\section{Cloud Server}

The sensor's data can be used in predicting future ailments and for sending periodical alerts to the medical practitioner. Fig 1 explains the sensor placed in the board.

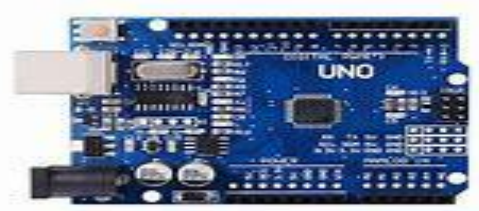

Fig. 1 Sensor board

\section{SYSTEM MODEL}

A gadget design or frameworks structure is the theoretical rendition that characterizes the shape, conduct, and more points of view of a gadget. A design depiction is a formal portrayal and portrayal of a device, arranged such that helps thinking roughly the frameworks and practices of the framework.

The structure graph of a framework wherein the principle components of capacities are spoken to through squares connected with the guide of the lines that show the connections of the squares. The design chart is commonly utilized for a higher stage, substantially less explicit depiction pointed more noteworthy at data the general standards and less at learning the data of execution. It recommends the relationship among particular segments of a framework. Framework structure can involve gadget parts, the remotely unmistakable living arrangements of those segments, the connections among them.

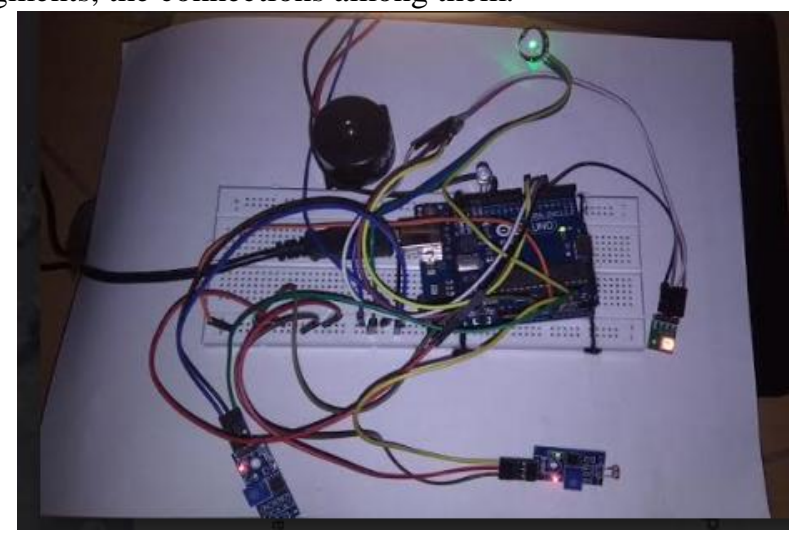

Fig. 2 System model

Fig 2 explains the system model of architecture diagram. It is demonstrated the how the system works.

\section{V.SYSTEM ARCHITECTURE}

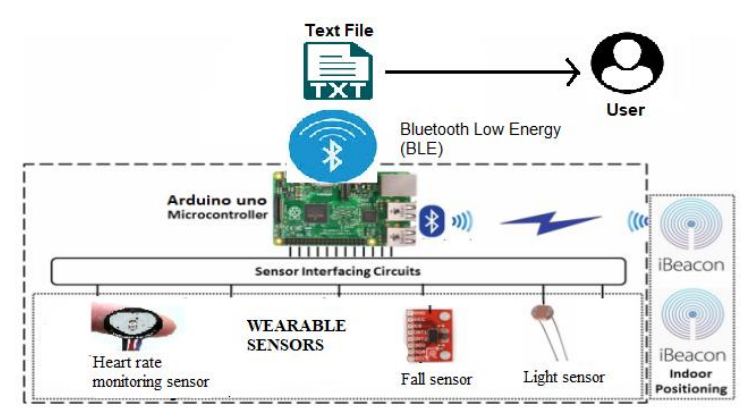

Fig. 3 System architecture

A framework's engineering can give a plan from which items might be secured, and frameworks progressed, so as to canvases together to place in power the general machine.

We present a framework that show the individuals by method for the utilization of a wearable gloves with gathered sensors. It obtain the wished records of the client and impart such information remotely the utilization of Bluetooth Low vitality .IBeacon transmits a Bluetooth low quality sign to conclude the presence inside its vicinity.The amassed actualities is spared and prepared inside the database. The measurements is as contrasted and the edge esteems and the remarks is despatched to the shopper.

\section{RESULT AND DISCUSSION}

As a proof of idea, a model framework has been actualized to assess the proposed IoT dynamic and helped living medicinal services framework. The framework has been con-figured to gather information from all the recorded sensors. In any case, in what pursues, the framework just 
introduces the information of the indoor situating, the fall location, and the light sensor. The readings of the other wearable sensors have been approved in the lab too. The framework has the client strolling for a couple of moments at that point suddenly fall on the ground, to copy certifiable situation. At the point when, the client tumbles down, there was a little change in speeding up in $x$-and y-pivot, and a critical spike in the quickening of $z$-hub exhibiting that the proposed framework can recognize unexpected falls, as material in medicinal services segment. The framework is conveyed a few iBeacon transmitters in the structure where the trial was led.

\section{CONCLUSION}

In this paper, the framework is displayed a low-control wearable IoT framework for dynamic and helped living medicinal services applications. We have laid out the primary parts of the proposed framework and clarified their execution subtleties. We have constructed a model to delineate the diverse presentation parts of the proposed framework. The primer execution assessment results have shown the effectiveness of the proposed framework - in spite of being an ease one. This makes the proposed framework a decent contender for executing a wide arrangement of wearable human services frameworks. Our future work will incorporate how to verify the entrance of the information and will build up a portable application that permits access of the information on handheld gadgets.

\section{REFERENCES}

1. S. Jaiswal 2017, 'Survey of Health Monitoring Management Using Internet of Things (IOT),' Int. J. Sci. Res., vol. 5, no. 11, pp. 2243-2246.

2. D. Kajaree 2017, 'A Survey on Healthcare Monitoring System Using Body Sensor Network', Int. J. Innov. Res. Comput.Commun. Eng., vol. 5, no. 2, pp. 1302-1309. 